\title{
Material Discrimination Algorithm Based on Hyperspectral Image
}

\author{
Jian Zhou $\mathbb{D}^{1},{ }^{1}$ Zhuping Wang, ${ }^{2}$ Yingjie Jiao, ${ }^{1}$ and Cong Nie $\mathbb{D}^{1}$ \\ ${ }^{1} X i$ 'an Modern Control Technology Research Institute, Xi'an, China \\ ${ }^{2} X i$ 'an Institute of Electromechanical Information Technology, Xi'an, China \\ Correspondence should be addressed to Cong Nie; abbqq_15986@163.com
}

Received 16 July 2021; Revised 18 August 2021; Accepted 24 August 2021; Published 13 September 2021

Academic Editor: Yi-Zhang Jiang

Copyright (c) 2021 Jian Zhou et al. This is an open access article distributed under the Creative Commons Attribution License, which permits unrestricted use, distribution, and reproduction in any medium, provided the original work is properly cited.

Hyperspectral information can be used to express the material properties of objects, which has a strong effect on camouflage recognition. However, it is difficult to process it directly because of the huge hyperspectral image data. Therefore, this paper proposes a new band selection algorithm to achieve band selection by simulating visual perception. The subspace clustering selfattention adversarial network is constructed to realize the initial selection of band. According to the visual chromatic aberration principle, a model is constructed to determine the band that combines the strongest response intensity of a particular material, and then this band is selected as the final band, therefore realizing the algorithm of material demarcation in this way.

\section{Introduction}

Visible near-infrared band images are obtained by sensors through detecting the electromagnetic radiation reflection of objects. It can precisely characterize ground objects so that each object has a spectral fingerprint which is of great significance to the identification of object materials $[1,2]$. However, a hyperspectral image has high spectral dimension and spatial resolution, so it is difficult to process it directly because of a large amount of data $[3,4]$. Thus, more in-depth studies have been carried out: in 2010, Yang et al. [5] used a supervised way to select band signals; Di et al. [6] applied a band selection to human face recognition and achieved good results. In 2010, Li and Qian [7] constructed a sparse matrix to analyze different bands; Samadzadegan and Mahmoudi [8] constructed the swarm intelligence to optimize band selection strategy. In 2012, Du et al. [9] established a collaborative sparse model to select hyperspectral bands; Hedjam and Cheriet [10] realized a band selection based on graph clustering. In 2013, Feng et.al [11] realized the band selection based on trivariate mutual information and clonal selection. Nakamura et al. [12] proposed a nature-inspired framework for band selection. In 2014, Su et al. [13] used the particle swarm optimization to optimize the band selection process; Xiurui Geng et al. [14] realized a band selection through gradient analysis of different band images. In 2015, Jia et al. [15] proposed a band selection scheme based on the idea of sorting; Patra et al. [16] introduced the idea of rough set to select bands. In 2016, Feng et al. [17] utilized the multiple kernel learning based on discriminative kernel clustering for hyperspectral band selection; Liu et al. [18] proposed a band selection algorithm based on the distribution of adjacent pixels. In 2017, Cao et al. [19] improved a classification map algorithm for fast hyperspectral selection; Shah et al. [20] proposed an algorithm of the dynamic frequency domain to realize band selection. In 2018, Wang et al. [21] proposed the optimal clustering framework to achieve hyperspectral band selection; Xie et al. [22] made modeling and analysis according to the representativeness of the bands. In 2019, Sun et al. [23] used a weighted kernel regulation to realize band selection. Sun et al. [24] calculated the variance between spectral bands and built a model for band selection. In 2020, Torres et al. [25] applied a band selection into the field of signal enhancement. Sun et al. [26] used the idea of low rank to cluster hyperspectral bands. Patra and Barman [27] focused on the image boundary intensity to realize band selection based on the fuzzy set.

To sum up, main problems of hyperspectral band selection are as follows. (1) It is difficult to establish a unified band selection model due to high dimensions of 
hyperspectral data. (2) The quality of band selection cannot directly show its effect. Therefore, according to the above problems, (1) a hyperspectral band selection algorithm is constructed based on vision and (2) a subspace clustering framework is proposed based on deep adversary for realizing the preliminary clustering of spectral information. A perception model based on color is proposed to visualize the difference between the target and the background to show the perception effect.

\section{A Visual Perception Algorithm}

More than $80 \%$ information is obtained by human vision. One object can be recognized and distinguished from the background mainly by the color. At present, the captured natural images can be regarded as the superposition of RGB three channels. On this basis, a large number of research studies on target extraction, image retrieval, and analysis have been carried out, and a series of achievements have been achieved. The natural images can be regarded as hyperspectral data with low number of channels. Therefore, we migrate the related algorithms of RGB images to the hyperspectral field, and the process is shown in Figure 1. (1) The subspace clustering network of deep countermeasure is constructed to realize the initial band selection. (2) According to the color difference of vision principle, a model is constructed to determine the band combination with the strongest response intensity of a specific material, and then the band is selected as the final band.

\subsection{Subspace Clustering Based on Self-Attention Adversarial.} When high-dimensional data are encoded to output lowdimensional feature representation, a large amount of information will be lost. However, the attention model, which is based on encoder-decoder framework, can lose less information.

$$
\operatorname{Attention}(Q, K, V)=s\left(Q, K^{T}\right) V
$$

where $S(\cdot)$ is the similarity function and $Q$ is the output information. The self-attention adversarial model we built is structured as Figure 2.

For true sample acquisition, $k$ groups of $A=\left\{A_{1}, A_{2}, \ldots\right.$, $\left.A_{k}\right\}$ are obtained through the similarity matrix learned by word expression layer. the projection residual from $A_{i}$ to the corresponding subspace $S_{i}$ is calculated as follows:

$$
L_{r}\left(Z_{i}\right)=\left\|Z_{i}-V_{i} V_{i}^{T} Z_{i}\right\|_{2}^{2}
$$

where $Z$ is the characteristic matrix, $V_{i}$ is the projection matrix, $T$ represents matrix transpose, and $L_{R}$ represents the projection residual. $m$ data with small residuals are selected as positive samples. The corresponding generator resistance loss function is as follows:

$$
L_{g}=\frac{1}{k m} \sum_{i=1}^{k} \sum_{j=1}^{m} L_{r}\left(\bar{Z}_{j}\right) .
$$

For false samples, the sampling layer randomly samples from the estimated subspace $S_{i}$ to generate $m$ false samples $\bar{Z}_{j}=\sum \theta_{j} Z_{j}$. In order to make the generated data closer to the learning subspace of discriminator, the antiloss is introduced to revise the existing loss function:

$$
L_{G}(\mathbf{C})=L_{g}+\frac{\lambda}{2}\|X-\widehat{X}\|^{2}+\frac{\alpha}{2}\|U-U C\|^{2}+\frac{\beta}{2}\|C\|_{p},
$$

where $\lambda$ is the balanced sparse.

A discriminator is constructed by projection residuals to distinguish true and false samples, and the probability loss function of samples belonging to subspace is established.

$$
L_{d}=\frac{1}{m} \sum_{j=1}^{m}\left\{L_{r}\left(Z_{j}\right)+\max \left(0, \varepsilon-L_{r}\left(Z_{j}\right)\right)\right\},
$$

where $\varepsilon$ is the parameter. The loss function of the discriminator corresponding to $k$-rent is as follows:

$$
L_{d}=\min _{V_{1}, V_{2}, \ldots, V_{k}} \frac{1}{k} \sum_{i=1}^{k} L_{d} .
$$

The second term of the following formula is introduced to increase the separation of different groups of subspaces after introducing regular terms. The third term of the following formula is to reduce $V_{i}$ :

$$
L_{E}=L_{d}+\mu_{1} \sum_{i \neq j}\left\|V_{i}^{T} V_{j}\right\|_{F}^{2}+\mu_{2} \sum_{i=1}^{k}\left\|V_{i}^{T} V_{i}-I\right\|_{F}^{2},
$$

where $\mu_{1}$ and $\mu_{2}$ are two constants greater than 0 .

In order to make better use of the local manifold structure information of the image, Laplacian regularization term is introduced into the loss function of the generator to construct the image connection relationship. The weight can be expressed as follows:

$$
W_{i j}= \begin{cases}\exp \left(\frac{\left\|x_{i}-x_{j}\right\|}{2 \sigma^{2}}\right), & x_{i}, x_{j} \in N_{k} \\ 0, & \text { others }\end{cases}
$$

where $N_{k}$ is the $K$ neighborhood of $n$ vertices. For the nonlinear manifold structure, the energy function is defined as follows:

$$
Q=\min _{q} \sum_{i, j=1}^{n}\left\|q\left(x_{i}\right)-q\left(x_{j}\right)\right\|^{2} W_{i j}
$$

According to the definition of the Laplace matrix,

$$
L=D-W\left(\text { s.t. } D_{i i}=\sum_{j} W_{i j}\right) \text {. }
$$

$Q$ can be rewritten as

$$
\begin{aligned}
& Q=\min _{q} \operatorname{tr}\left(\bar{Q} L \bar{Q}^{T}\right), \\
& \bar{Q}=\left[q\left(x_{1}\right), q\left(x_{2}\right), \ldots, q\left(x_{n}\right)\right] .
\end{aligned}
$$




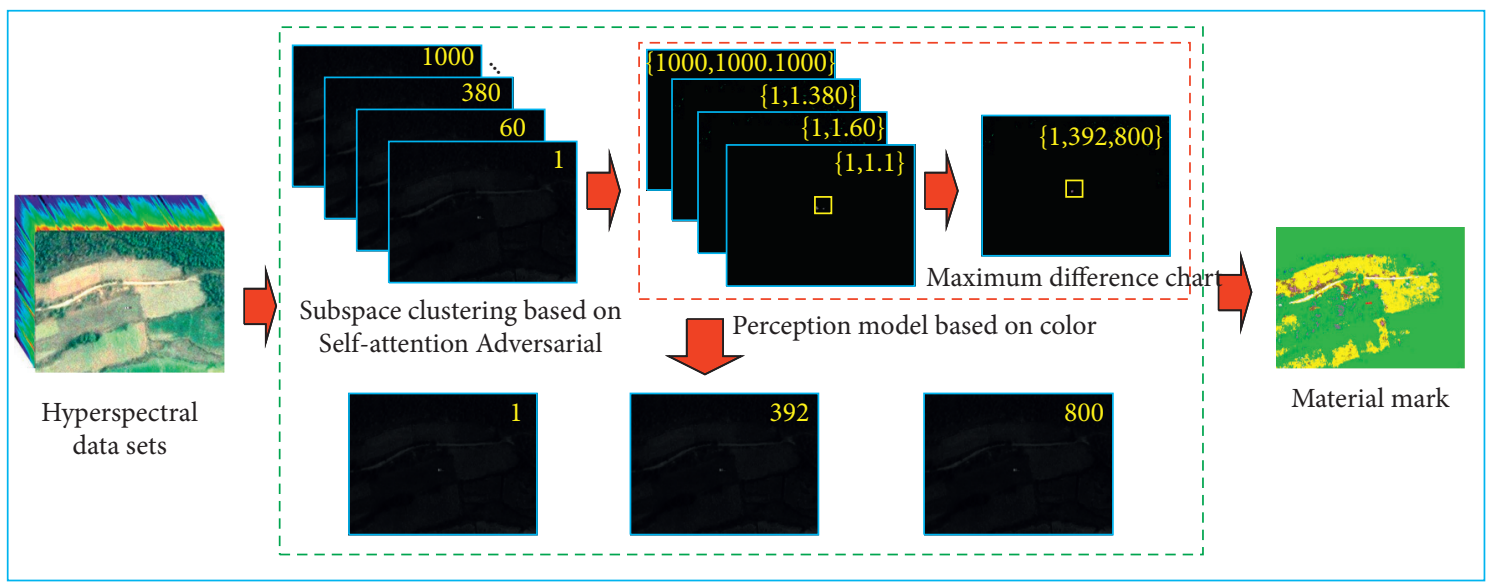

Figure 1: Flow chart of the band selection algorithm.

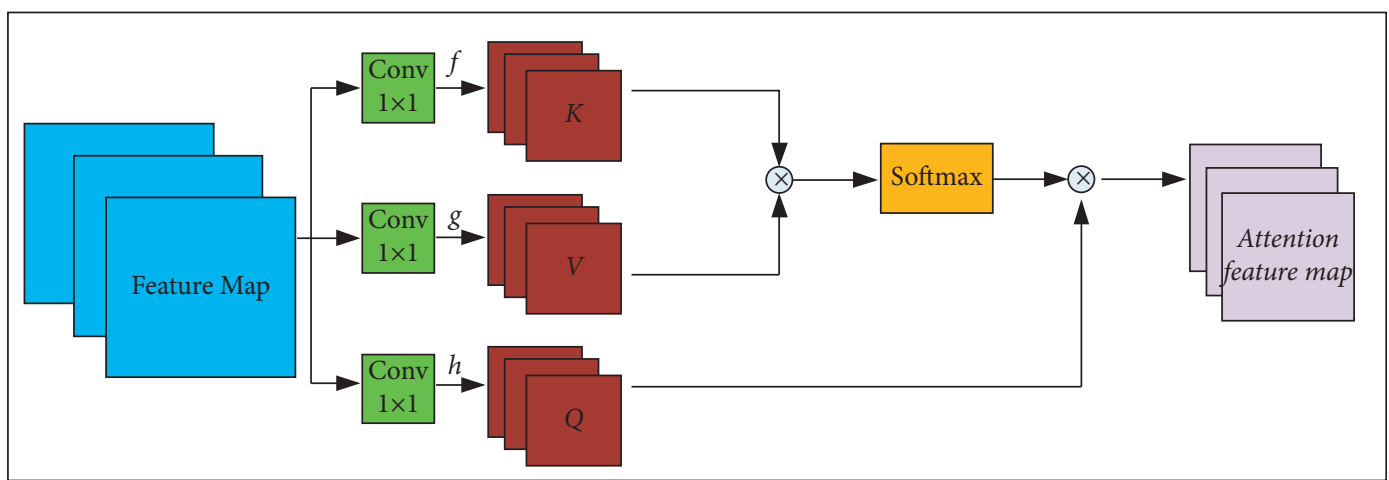

FIGURE 2: Self-attention adversarial network.

The final generator loss function is obtained as follows:

$$
L_{G}(C)=L_{g}+\frac{1}{2}\left\{\lambda\|X-\hat{X}\|^{2}+\alpha\|U-U C\|^{2}+\beta\|C\|_{p}+\gamma \operatorname{tr}\left(C L C^{T}\right)\right\} .
$$

2.2. Perception Model Based on Color. According to Gestalt psychocognitive analysis, objects can be recognized, mainly by the eyes and brain. When the eyes observe the images, they cluster themselves according to certain rules to make them become a comprehensible structural entity. Among them, color feature is an effective way.
On the basis of the previous analysis in the last section, in order to more comprehensively express the spectral information between bands, a model is established on the basis of relative entropy:

$$
\begin{aligned}
S(X, Y) & =D_{\mathrm{KL}}(P \mid Q)-\lambda M_{t}(X, Y), \\
D_{\mathrm{KL}}(P \mid Q) & =\sum P(x) \ln \frac{P(x)}{Q(x)}, \quad P(x), Q(x) \in[0,1], \\
M_{t} & =\left[\begin{array}{c}
m\left(X_{1}, X_{1}\right) \ldots m\left(X_{1}, X_{M}\right) \\
\ldots \\
m\left(X_{M}, X_{1}\right) \ldots m\left(X_{M}, X_{M}\right)
\end{array}\right], \\
m(X, Y) & =\sum_{i=1}^{n} \sum_{j=1}^{m} p\left(x_{i} y_{j}\right) \log _{2} \frac{p\left(x_{i} / y_{j}\right)}{p\left(x_{i}\right)},
\end{aligned}
$$


where $S(X, Y)$ is the spectral correlation between band $X$ and band $Y ; D_{\mathrm{KL}}(P \mid Q)$ is the relative entropy of probability distribution corresponding to band $X$ and band $Y$; and $M_{t}$ $(X, Y)$ is the average mutual information between band $X$ and band $y . \lambda$ is the weight coefficient, which is determined by the relative amplitude of $D_{\mathrm{KL}}(P \mid Q)$ and $M_{t}(X, Y)$.

In order to better integrate the entropy information into it, we modify $S(X, Y)$. Because the entropy divergence and the average mutual information are basically equally important to the band selection process, in order to make the contribution of the corresponding matrix $M_{\mathrm{KL}}$ and $M_{t}$ consistent to $\mathrm{S}$, the normalization function is constructed.

$$
\begin{aligned}
S(X, Y) & =C \times M_{\mathrm{KL}}-M_{t}, \\
M_{\mathrm{KL}} & =\left[\begin{array}{c}
0 \ldots D_{\mathrm{KL}}\left(X_{1} \mid X_{M}\right) \\
\ldots \\
D_{\mathrm{KL}}\left(X_{M} \mid X_{1}\right) \ldots 0
\end{array}\right], \\
C & =\frac{\sum_{i=1}^{M} \sum_{j=1}^{M}\left|M_{t}(i, j)\right|}{\sum_{i=1}^{M} \sum_{j=1}^{M}\left|M_{K L}(i, j)\right|}
\end{aligned}
$$

Different pixel values may correspond to the same color name. For this purpose, we construct the mapping relation; given the data $D=\left\{d_{1}, \ldots, d_{N}\right\}$, the corresponding word is $W=\left\{w_{1}, \ldots, w_{M}\right\}$; these words are considered from the potential theme $Z=\left\{z_{1}, \ldots, z_{K}\right\}$. Therefore, a probability model is constructed as follows:

$$
p(w \mid d)=\sum_{z \in Z} p(w \mid z) p(z \mid d),
$$

where $p(w \mid z)$ and $p(z \mid d)$ are prior probabilities. The EM algorithm is used to estimate the maximum similarity as follows:

$$
L=\sum_{d \in D} \sum_{w \in W} n(d, w) \log \{p(d) p(w \mid d)\}
$$

where $n(d, w)$ is the frequency of occurrence. Through training, we get the following results:

$$
\begin{aligned}
p(z \mid w, d) & \longleftarrow \theta_{d}(z) \phi_{z}(w), \\
\phi_{z}(w) & \longleftarrow \sum_{d} n(d, w) p(z \mid w, d), \\
\theta_{d}(z) & \longleftarrow(\alpha-1)+\sum_{w} n(d, w) p(z \mid w, d),
\end{aligned}
$$

where $\alpha$ is a parameter. The corresponding maximum similarity can be written as follows:

$$
L=\sum_{d \in D} \sum_{w \in W} n(d, w) \log (d, w)-\gamma \sum_{z \in Z} \sum_{w \in W}(p(z \mid w)-\rho(w))^{2} .
$$

Based on this, the color mapping is realized, and the colors are sorted according to Figure 3 to construct the differentiation degree.

\section{Experiment Result and Analysis}

The experiment is composed of visible infrared hyperspectral data and software simulation data [28], as shown in Figure 4, including grassland, sand, vehicles, buildings, and other typical targets. It can be seen from the figure that the pixel values displayed by different ground objects in different bands are different, and there are also differences in the pixel values of ground objects in the same band, which is the basis of band selection. We normalized the hyperspectral images to $512 \times 512 \times 300$.

3.1. Display of Spectral Curves of Typical Ground Objects. In order to show the spectral curves of typical ground objects, the spectral curves of leaves and sky are selected to display, as shown in Figure 5. The horizontal axis represents the band number and the vertical axis represents the pixel value. It can be seen that the same kind of features has strong similarity, and different types of features have differences. Although the internal targets have volatility, the overall volatility is small. In the areas of leaves and sky, the most significant area is concentrated in the $0-300$ band. In the area of sky, the pixel value of $0-300$ band reaches saturation state. Based on the above analysis, the sky and leaves can be effectively distinguished.

3.2. Band Clustering Effect. In order to verify the accuracy of moving target extraction, we introduce the OA [29] overall accuracy and kappa coefficient. OA represents the proportion of samples with correct classification to all samples. Kappa is an index used for the consistency test [30]:

$$
\begin{aligned}
\mathrm{OA} & =\frac{1}{N} \sum_{i=1}^{r} x_{i i}, \\
\text { kappa } & =\frac{N \sum_{i=1}^{r} x_{i i}-\sum_{i=1}^{r}\left(x_{i+} \times x_{+i}\right)}{N^{2}-\sum_{i=1}^{r}\left(x_{i+} \times x_{+i}\right)} .
\end{aligned}
$$

The band selection effect of real scene hyperspectral image is shown in Figure 6, and the band selection effect of simulated scene hyperspectral image is shown in Figure 7. It can be seen from the figure that the effect of the real image is slightly lower than that of the simulated image, which is due to the stable noise and spectral curve contained in the simulated image. However, the information contained in the real image is more complex and has a certain volatility.

The sparse nonnegative matrix factorization (SNMF) algorithm [6] transforms the problem of band selection into the problem of sparse decomposition, and it has a certain effect to extract significant spectral images. The fast volume gradient (FVG) algorithm [13] establishes the model according to the gradient to realize the band selection, and the effect is better for the region with obvious boundary. The variable precision neighborhood (VPN) algorithm [17] constructs fuzzy sets according to the relationship between adjacent pixels to realize band selection. The fast and late low rank (FLLR) algorithm [20] introduces the idea of low rank to calculate the redundancy between bands and realize band 


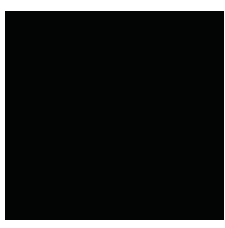

1 black

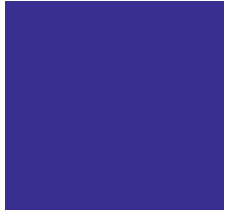

6 siniy

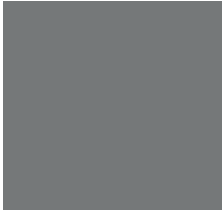

2 grey

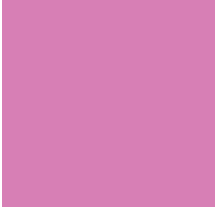

7 violet

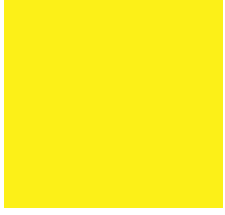

3 yellow

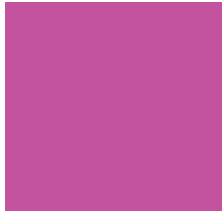

8 magenta

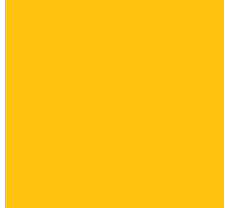

4 gold

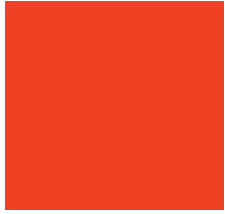

9 red

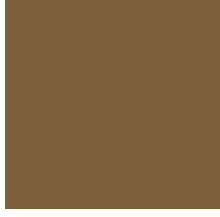

5 olive

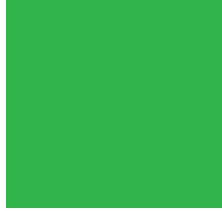

10 green

FIGURE 3: Mapping color sequence graph.

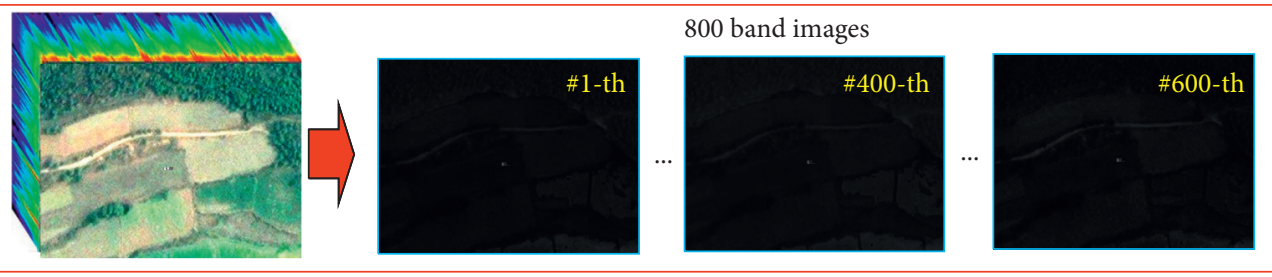

(a)

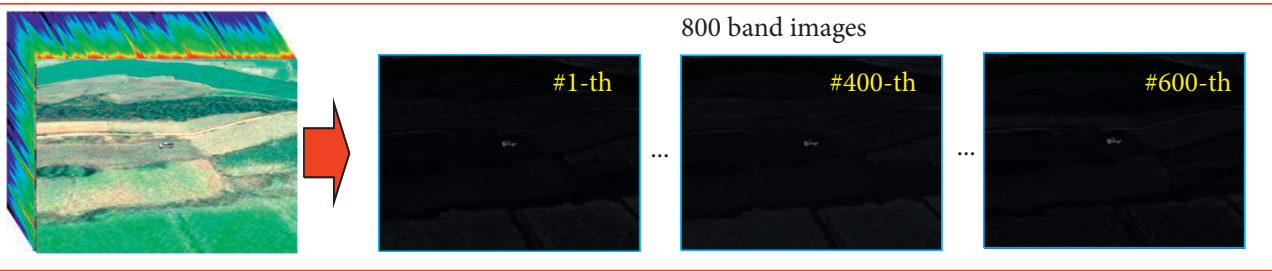

(b)

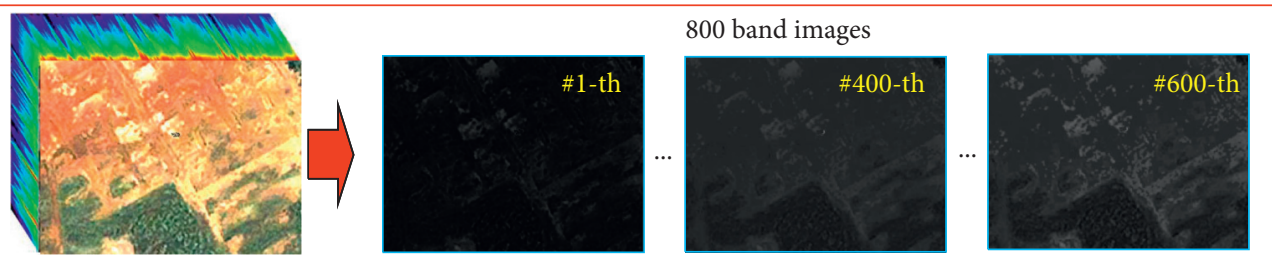

(c)

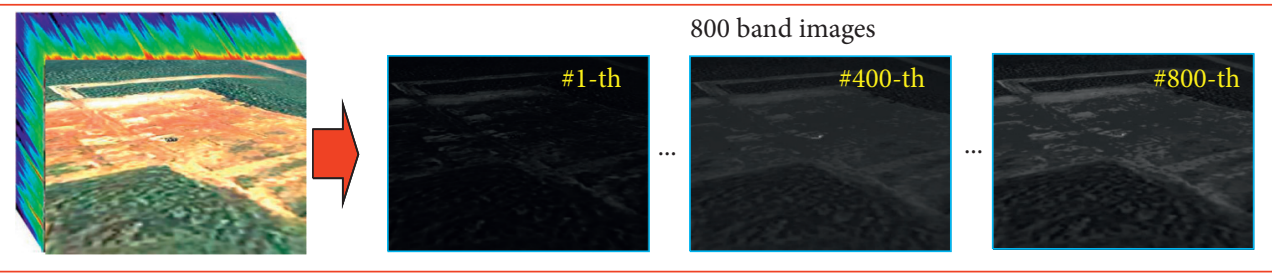

(d)
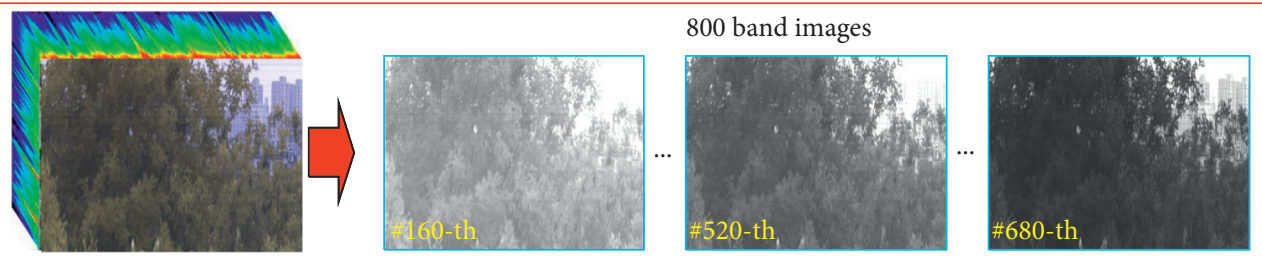

(e)

FIGURE 4: Data display: (a) simulated overlook image sequence; (b) simulated sideview image sequence; (c) simulated overlook image sequence; (d) simulated sideview image sequence; (e) real image sequence. 


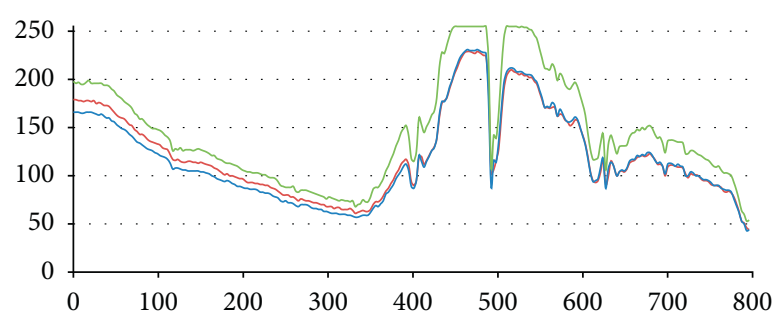

(a)

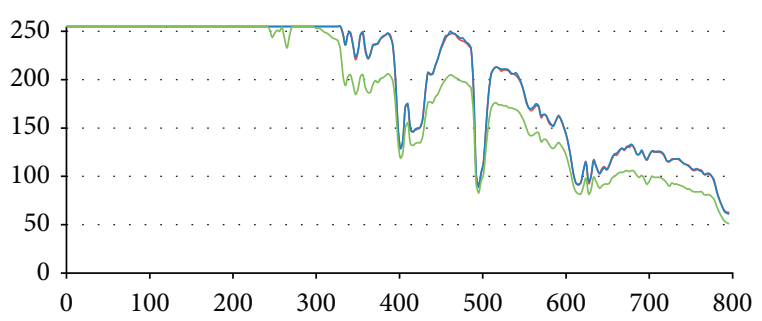

(b)

FIGURE 5: Display of spectral curves of typical ground objects: (a) spectral curve of leaves; (b) spectral curve of sky.

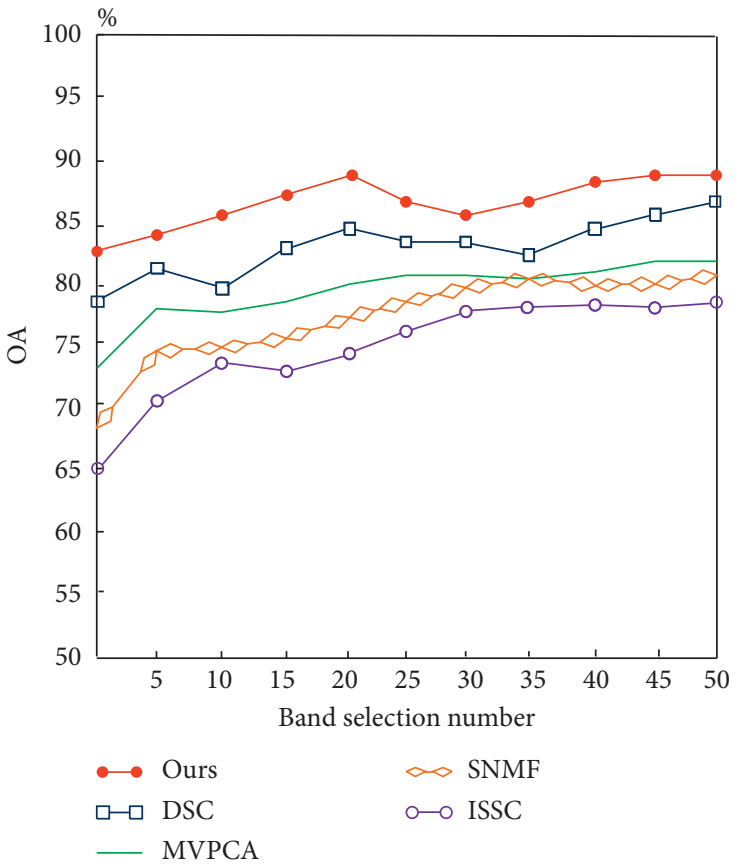

(a)

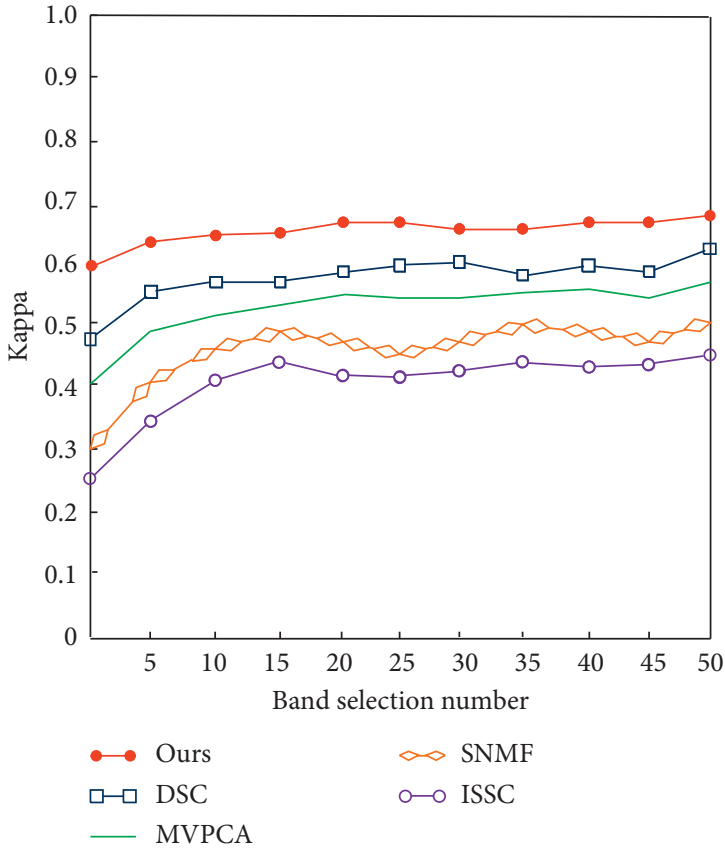

(b)

FIgURE 6: Experimental results of real hyperspectral sequences: (a) OA; (b) kappa.

selection. In this paper, a subspace clustering algorithm is proposed based on depth confrontation, which fully considers the correlation of bands and optimizes the loss function to achieve band selection. On the real data set, OA reaches $90 \%$ and kappa reaches 0.67 . OA and kappa are $96 \%$ and 0.92 , respectively, in the simulation data set, which reach better results.

3.3. Target Detection Effect. On the basis of the optimal band selection, different target material clustering algorithms are used for comparison. The detection results of real data and simulated data are shown in Figure 8. Hu et al. [31] proposed the SVM algorithm to extract image features for clustering to achieve enhancement. Han et al. [32] constructed CNN to extract target features. Shi and Pun [33] built multiscale RESNET to realize target detection. Li et al. [34] detected targets based on boundary features. The above algorithms analyze the target from the perspective of morphology to achieve target detection. Based on clustering, in this paper, we construct a visual perception model to detect the target and use the difference of visual mapping to measure the detection rate of the target, which has a good effect, and the ROC curve value is the highest.

The proposed algorithm constructs the mapping model of visual perception by fusing images with three bands. The mapping results of real data using different band combinations are shown in Figure 9(a). \{0,38, 187 $\}$ segment maps the leaf region to red, but it cannot distinguish the building and sky areas effectively. $\{1,161,35\}$ spectrum segment can distinguish the building area from other areas, which verifies the effectiveness of the proposed algorithm. The mapping results of simulated data using different band combinations are shown in Figure 9(b). \{0.4, 8.0, 12\} spectrum can distinguish grassland from land but cannot distinguish grassland from vehicle. $\{0.6,0.6,10.4\}$ bands can extract vehicles effectively and suppress grassland and land areas. The effectiveness of the proposed algorithm is verified. 


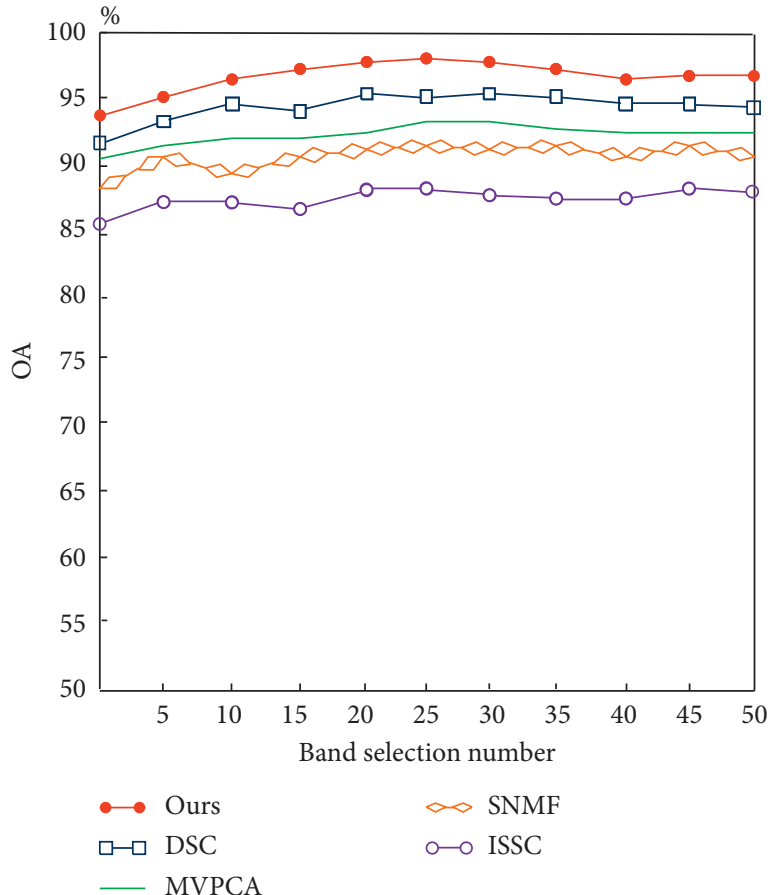

(a)

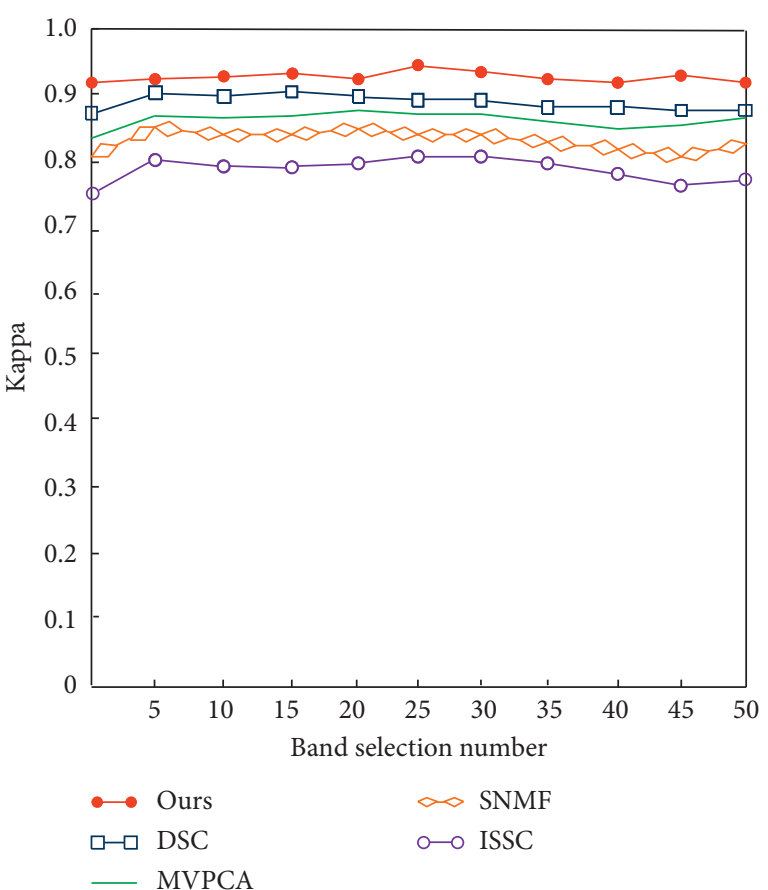

(b)

FIGURE 7: Experimental results of simulated hyperspectral sequences: (a) OA; (b) kappa.

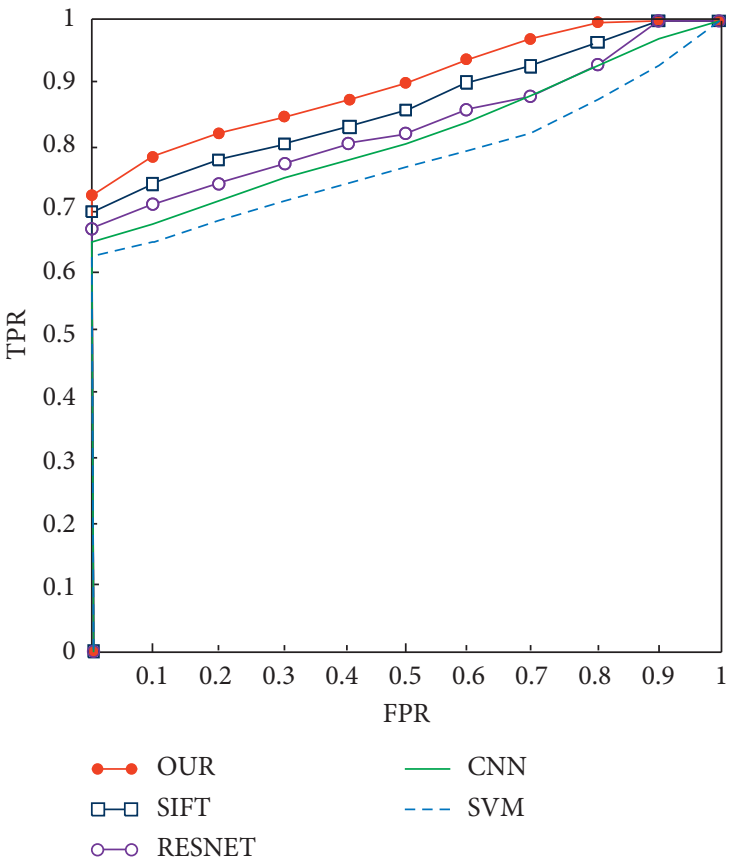

(a)

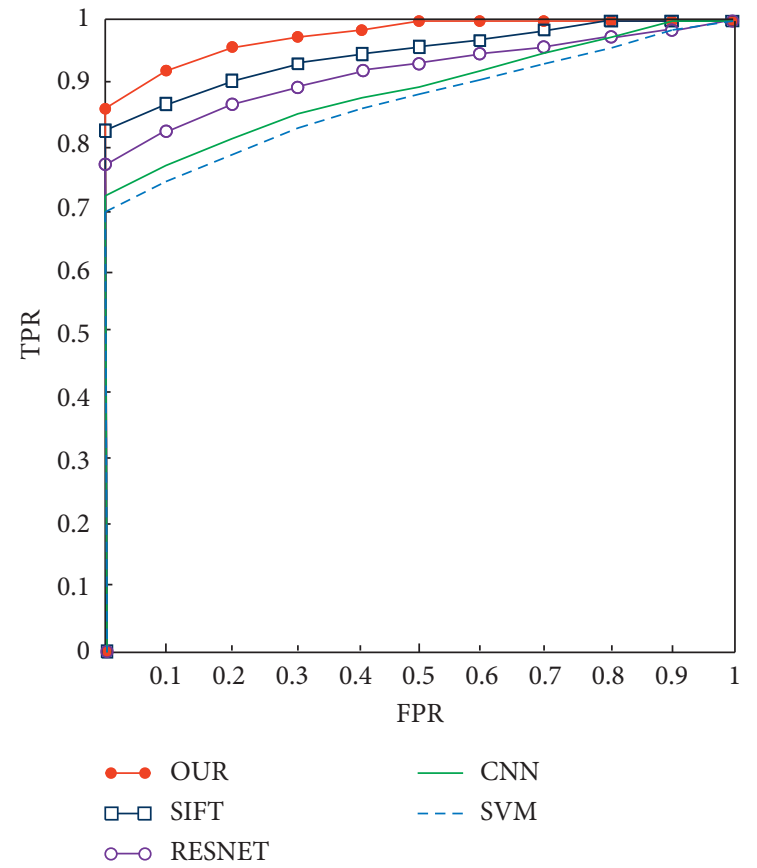

(b)

Figure 8: Roc curve: (a) real data; (b) simulated data. 


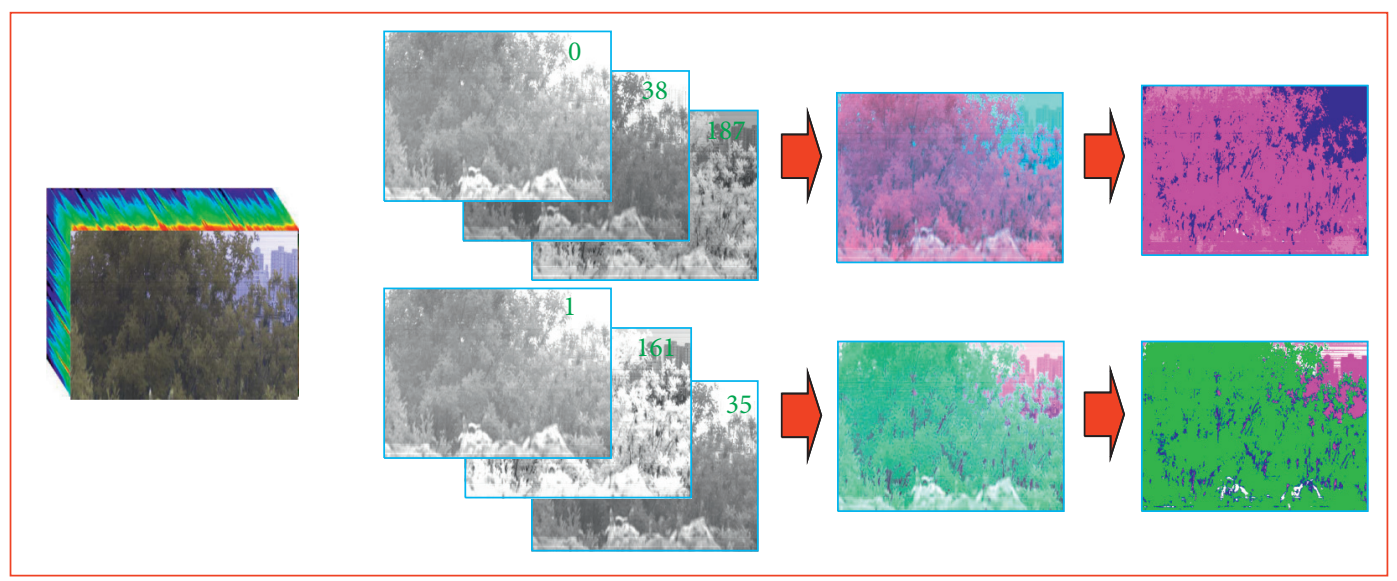

(a)

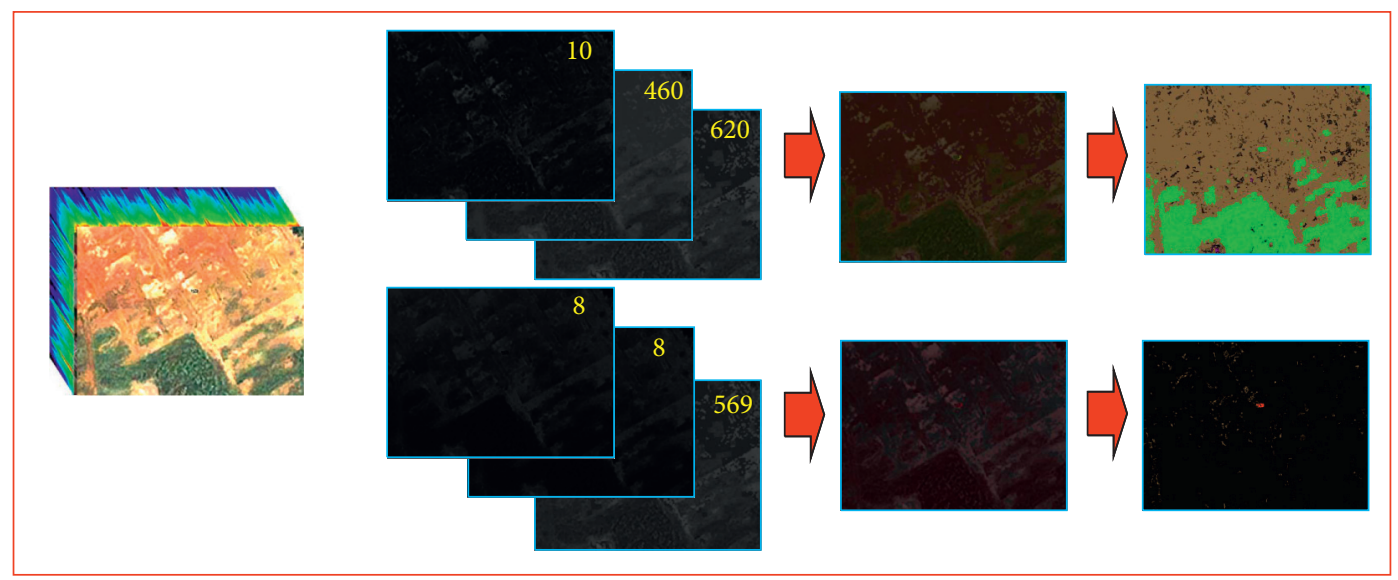

(b)

Figure 9: Target detection experimental results: (a) real data; (b) simulated data.

\section{Conclusion}

Hyperspectral images have spatial resolution and interspectral resolution, which plays an important role in material recognition. Aiming at the difficulty of hyperspectral band selection, a deep adversarial subspace clustering network is constructed to select the representative band, which can select a representative band. From the perspective of psychology, a color perception model is constructed to highlight the significant areas. Experiments show that the proposed algorithm has good results. On this basis, it can carry out material recognition of typical targets and hidden targets.

\section{Data Availability}

This paper experimented with two databases. One is from https://figshare.com/articles/dataset/Main_zip/2007723/3. The other is the real hyperspectral database. The data are too large and can be obtained from the corresponding author upon request. The database is for scientific research only.

\section{Conflicts of Interest}

The authors declare that they have no conflicts of interest.

\section{Acknowledgments}

This work was supported by Postdoctoral Science Foundation of China under grant no. 2020M682149.

\section{References}

[1] V. K. Venugopal, K. Vaidhya, M. Murugavel et al., "Unboxing AI - radiological insights into a deep neural network for lung nodule characterization," Academic Radiology, vol. 27, no. 1, pp. 88-95, 2020.

[2] S. Sawant and M. Prabukumar, "A survey of band selection techniques for hyperspectral image classification," Journal of Spectral Imaging, vol. 9, 2020.

[3] C. Chang, Y. Kuo, S. Chen, C. C. Liang, K. Y. Ma, and P. F. Hu, "Self-mutual information-based band selection for hyperspectral image classification," IEEE Transactions on Geoscience and Remote Sensing, vol. 59, 2020.

[4] W. Sun, J. Peng, G. Yang, and Q. Du, "Fast and latent lowrank subspace clustering for hyperspectral band selection," IEEE Transactions on Geoscience and Remote Sensing, vol. 58, no. 6, pp. 3906-3915, 2020.

[5] H. Yang, Q. Du, H. Su, and Y. Sheng, "An efficient method for supervised hyperspectral band selection," IEEE Geoscience and Remote Sensing Letters, vol. 8, no. 1, pp. 138-142, 2010. 
[6] W. Di, L. Zhang, D. Zhang, and Q. Pan, "Studies on hyperspectral face recognition in visible spectrum with feature band selection," IEEE Transactions on Systems, Man, and Cybernetics - Part A: Systems and Humans, vol. 40, no. 6, pp. 1354-1361, 2010.

[7] J.-m. Li and Y.-t. Qian, "Clustering-based hyperspectral band selection using sparse nonnegative matrix factorization," Journal of Zhejiang University - Science C, vol. 12, no. 7, pp. 542-549, 2011.

[8] F. Samadzadegan and F. Mahmoudi, "Optimum band selection in hyperspectral imagery using swarm intelligence optimization algorithms," in Proceedings of the 2011 International Conference on Image Information Processing, pp. 1-6, IEEE, Shimla, India, November 2011.

[9] Q. Du, J. Bioucas-Dias, and A. Plaza, "Hyperspectral band selection using a collaborative sparse model," in Proceedings of the IEEE International Geoscience and Remote Sensing Symposium, pp. 3054-3057, IEEE, Munich, Germany, July 2012.

[10] R. Hedjam and M. Cheriet, "Hyperspectral band selection based on graph clustering," in Proceedings of the 2012 11th International Conference on Information Science, Signal Processing and Their Applications (ISSPA), pp. 813-817, Montreal, Canada, July 2012.

[11] J. Feng, L. Jiao, X. Zhang, and T. Sun, "Hyperspectral band selection based on trivariate mutual information and clonal selection," IEEE Transactions on Geoscience and Remote Sensing, vol. 52, no. 7, pp. 4092-4105, 2013.

[12] R. Nakamura, L. Fonseca, J. Dos Santos, R. d. S. Torres, X. Yang, and J. P. Papa, "Nature-inspired framework for hyperspectral band selection," IEEE Transactions on Geoscience and Remote Sensing, vol. 52, no. 4, pp. 2126-2137, 2013.

[13] H. Su, Q. Du, G. Chen, and P. Du, “Optimized hyperspectral band selection using particle swarm optimization," IEEE Journal of Selected Topics in Applied Earth Observations and Remote Sensing, vol. 7, no. 6, pp. 2659-2670, 2014.

[14] X. Xiurui Geng, K. Kang Sun, L. Luyan Ji, and Yongchao Zhao, "A fast volume-gradient-based band selection method for hyperspectral image," IEEE Transactions on Geoscience and Remote Sensing, vol. 52, no. 11, pp. 7111-7119, 2014.

[15] S. Jia, G. Tang, J. Zhu, and Q. Li, "A novel ranking-based clustering approach for hyperspectral band selection," IEEE Transactions on Geoscience and Remote Sensing, vol. 54, no. 1, pp. 88-102, 2015.

[16] S. Patra, P. Modi, and L. Bruzzone, "Hyperspectral band selection based on rough set," IEEE Transactions on Geoscience and Remote Sensing, vol. 53, no. 10, pp. 5495-5503, 2015.

[17] J. Feng, L. Jiao, T. Sun, H. Liu, and X. Zhang, "Multiple kernel learning based on discriminative kernel clustering for hyperspectral band selection," IEEE Transactions on Geoscience and Remote Sensing, vol. 54, no. 11, pp. 6516-6530, 2016.

[18] Y. Liu, H. Xie, L. Wang, and K. Tan, "Hyperspectral band selection based on a variable precision neighborhood rough set," Applied Optics, vol. 55, no. 3, pp. 462-472, 2016.

[19] X. Cao, C. Wei, J. Han, and L. Jiao, "Hyperspectral band selection using improved classification map," IEEE Geoscience and Remote Sensing Letters, vol. 14, no. 11, pp. 2147-2151, 2017.

[20] V. Shah, S. Bhattacharjee, S. Silvestri et al., "Designing sustainable smart connected communities using dynamic spectrum access via band selection," in Proceedings of the 4th ACM
International Conference on Systems for Energy-Efficient Built Environments, pp. 1-10, Delft, Netherlands, November 2017.

[21] Q. Wang, F. Zhang, and X. Li, "Optimal clustering framework for hyperspectral band selection," IEEE Transactions on Geoscience and Remote Sensing, vol. 56, no. 10, pp. 5910-5922, 2018.

[22] F. Xie, F. Li, C. Lei, and L. Ke, "Representative band selection for hyperspectral image classification," ISPRS International Journal of Geo-Information, vol. 7, no. 9, p. 338, 2018.

[23] W. Sun, G. Yang, J. Peng, and Q. Du, "Hyperspectral band selection using weighted kernel regularization," IEEE Journal of Selected Topics in Applied Earth Observations and Remote Sensing, vol. 12, no. 9, pp. 3665-3676, 2019.

[24] W. Sun, J. Peng, G. Yang, and Q. Du, "Correntropy-based sparse spectral clustering for hyperspectral band selection," IEEE Geoscience and Remote Sensing Letters, vol. 17, no. 3, pp. 484-488, 2019.

[25] R. M. Torres, P. W. T. Yuen, C. Yuan, J. Piper, C. McCullough, and P. Godfree, "Spatial spectral band selection for enhanced hyperspectral remote sensing classification applications," Journal of Imaging, vol. 6, no. 9, p. 87, 2020.

[26] W. Sun, G. Yang, J. Peng et al., "A multiscale spectral features graph fusion method for hyperspectral band selection," IEEE Transactions on Geoscience and Remote Sensing, pp. 1-12, 2021.

[27] S. Patra and B. Barman, "A novel dependency definition exploiting boundary samples in rough set theory for hyperspectral band selection," Applied Soft Computing, vol. 99, Article ID 106944, 2021.

[28] Pedram Ghamisi and Stuart Phinn, "Fusion of LiDAR and Hyperspectral Data," 2015.

[29] S. Qiu, K. Cheng, L. Cui, D. Zhou, and Q. Guo, "A moving vehicle tracking algorithm based on deep learning," Journal of Ambient Intelligence and Humanized Computing, pp. 1-7, 2020.

[30] S. Qiu, Y. Tang, Y. Du, and S. Yang, "The infrared moving target extraction and fast video reconstruction algorithm," Infrared Physics \& Technology, vol. 97, pp. 85-92, 2019.

[31] R. Hu, X. Zhu, Y. Zhu, and J. Gan, "Robust SVM with adaptive graph learning," World Wide Web, vol. 23, no. 3, pp. 1945-1968, 2020.

[32] M. Han, R. Cong, X. Li, H. Fu, and J. Lei, "Joint spatialspectral hyperspectral image classification based on convolutional neural network," Pattern Recognition Letters, vol. 130, pp. 38-45, 2020.

[33] C. Shi and C. M. Pun, "Multi-scale hierarchical recurrent neural networks for hyperspectral image classification," Neurocomputing, vol. 294, pp. 82-93, 2018.

[34] Y. Li, L. Fan, and W. Xie, "TGSIFT: robust SIFT descriptor based on tensor gradient for hyperspectral images," Chinese Journal of Electronics, vol. 29, no. 5, pp. 916-925, 2020. 